\title{
Solid matrices for fabrication of magnetic iron oxide nanocomposites: synthesis, properties, and application for the adsorption of heavy metal ions and dyes
}

\begin{abstract}
A massive release of harmful substances especially heavy metal ions and dyes to the environment has been a major concern due to many people disregards the proper protocols in the waste management. The freshwater supplies are threatened and huge discharge of pollutants result from various anthropogenic activities may pose a major threat to the living organisms and negatively affect the ecosystem stability. This article reviews the development of magnetic iron oxide nanocomposites for removal of heavy metal ions and dyes from water. The highlight will be focused on current research activities for controlled size and dispersion of magnetic iron oxide nanoparticles within solid matrices including zeolites, silica, clays, carbon, activated carbon, graphene and graphene oxide. The magnetic properties of the nanocomposites will be evaluated to determine whether they possessed sufficient magnetization for easy separation by an external magnet. The unique features of the synthesized materials will be investigated along with their application in the removal of heavy metal ions and dyes. The advantages and limitations of the magnetic nanocomposites will be highlighted to determine their adsorption ability. The effect of various parameters such as $\mathrm{pH}$, contaminants concentration, adsorbent dosage, contact time and temperature will be summarized to identify the best condition for effective pollutants removal..
\end{abstract}

Keyword: Magnetic iron oxide; Nanocomposites; Heavy metal ions; Dyes; Adsorption 